\title{
Acknowledgement of Reviewers 2018
}

Published online: 16 January 2019

(C) Springer Science+Business Media, LLC, part of Springer Nature 2019

The Editor, Eric Rowinsky, and the Associate Editors would like to acknowledge the valuable contributions of the reviewers to the journal. We fully understand the burden and effort that are involved in the reviews and are very grateful for your support. We rely on your expertise to maintain the standards of excellent science for the journal, Investigational New Drugs.

Ghassan Abou-Alfa
Eishi Ashihara
Paul Beavis
Jordan Berlin
Katarzyna Blazewska
Guido Bocci
John Bridgewater
Mans Broekgaarden
Elizabeth Buchbinder
Eric Chen
Qi Chen
Belamy Cheung
K.N. Chi
Gabriela Chiorean
Roger Cohen
Natalie Cook
Luciana Corrêa
Simon Crabb
Suman Dasgupta
Miranda de Jager
R. Donald Harvey
Dong-Jun Fu
Daichi Fujimoto
Weimin Gao
Beppe Giaccone
Sharlene Gill
M. Concepción Gimeno

Milena Gusella
Akinobu Hamada
Petra Heffeter
Howard Hochster
Satoshi Igawa
Hisao Imai
Rao Jaladanki
Li Jing
Nenad Joksimović
Subash C. Jonnalagadda
Masahi Kanai
Christos Kyriakopoulos
Rosamaria Lappano
Ismael Lares-Asseff
Chia-Chi (Josh) Lin
Jun Liu
Zhiqiang Liu
Jon Loree
Hideki Maeda
Stephen Mathew
Hans Minderman
Olivier Mir
Elena Morosova
Sumimasa Nagai
Pollyanna Oliveira
E.M. O’Reilly
Brian Piper

\author{
Alessandro Poggi \\ Edvin Pozharskiy \\ Antonino Puglisi \\ Qi Qi \\ Aramandla Ramesh \\ Mark Ratain \\ Stephan Joel Reshkin \\ Vincent Ribrag \\ Oliver Sartor \\ Tarah Satoh \\ Michael Sawyer \\ Sarah Schott \\ Desh Deepak Singh \\ Anatoly Skalny \\ John Spencer \\ Mitsukuni Suenaga \\ Masayuki Takeda \\ Motohiro Tamiya \\ Tsuimin Tsai \\ Chris Twelves \\ Ulka Vaishampayan \\ Hui-Chun Wang \\ Harvey Wong \\ Liu Wukun \\ Dmitry Zhdanov \\ Zongjian Zhu
}

Publisher's Note Springer Nature remains neutral with regard to jurisdictional claims in published maps and institutional affiliations. 